\title{
FACTORES ASOCIADOS A LA SUPERVIVENCIA DEL DIENTE CON ENDODONCIA EN PACIENTES MENORES DE 20 AÑOS ATENDIDOS EN UNA IPS PRIVADA, 2006-2012
}

\author{
Eliana Pineda Vélez ${ }^{*}$ Esp., MSc, Ángela María Segura, MSc, Ph.D. 2 \\ Facultad de Odontología, Universidad de Antioquia, Colombia \\ ${ }_{2}$ Grupo de Epidemiología y Bioestadística, Universidad CES, Colombia
}

Recibido: 11 de febrero del 2013. Aprobado: 8 de marzo del 2013.

*Autor de correspondencia: Eliana Pineda Vélez. Facultad de Odontología. Universidad de Antioquia, Medellín, Colombia, (57) 421967 72, Calle 64 52-59, correo electrónico: elipineda1@gmail.com

Cómo citar este artículo: Pineda Vélez E, Segura AM. Factores asociados a la supervivencia del diente con endodoncia en pacientes menores de 20 años atendidos en una IPS privada, 2006-2012. Revista Nacional de Odontología. 2013; 9(16): 43-50.

Resumen. Introducción: la endodoncia es la rama de la odontología que se ocupa del estudio de la morfología, la función, las alteraciones de la pulpa dental y la región periodontal, así como de su tratamiento. El éxito en endodoncia es elevado, pero la supervivencia se ha estudiado poco. El objetivo de esta investigación fue determinar el tiempo de supervivencia y cómo los factores demográficos y clínicos afectan la pérdida de dientes tratados con endodoncia. Métodos: se hizo un estudio de seguimiento a una cohorte con análisis de supervivencia, en el que se analizó el estado de los dientes tratados con endodoncia, a partir de la recolección sistemática de información, que se extrajo de las historias clínicas. Resultados: se encuentra una población con un ingreso bajo y con una educación técnica o profesional, y con predominio de los hombres; se presenta una velocidad de extracción o pérdida del diente mayor en los que tienen un nivel salarial bajo, y con lesiones apicales mayores de $11 \mathrm{~mm}$. Discusión: para una población específica menor de veinte años los resultados clínicos son similares a investigaciones publicadas en pacientes mayores a esta edad. Conclusión: en los dientes con lesiones mayores a $11 \mathrm{~mm}$ la pérdida dental es estadísticamente significativa.

Palabras clave: análisis multivariado, endodoncia, extracción dental, modelo de Cox, supervivencia.

Factors Associated to Survival of Endodontically-treated Teeth in Patients Younger than 20 Years of Age

Treated at a Private IPS in the Period 2006-2012

Abstract. Introduction: Endodontics is the branch of dentistry that studies the morphology, function and alterations of the dental pulp and periapical region. It also deals with the treatment of pulp and periapical pathologies. Although the success of endodontic procedures is high, the survival of endodontically treated teeth has been studied scarcely. The objective of this study was to determine the survival rate and the demographic and clinical factors that affect the loss of endodontically treated teeth. Methods: We conducted a cohort study with a survival analysis on endodontically treated teeth. Data was acquired from dental records. Results: In general, the studied population was characterized by a low income and educational status and was composed predominantly by males. Males lost more teeth at a faster rate than females and this phenomenon was affected by the low income mainly and by periapical lesions greater than $11 \mathrm{~mm}$. Discussion: For a specific population under 20 years of age the clinical results are similar to those of previous published research dealing with older patients. Conclusions: A rapid loss of teeth with endodontic treatment was significantly explained by the Presence of lesions exceeding $11 \mathrm{~mm}$ in diameter.

Keywords: multivariate analysis, endodontics, tooth extraction, Cox models, survival.
Fatores associados à sobrevivência do dente com endodontia em pacientes menores de 20 anos atendidos em uma IPs particular, 2006-2012

Resumo. Introdução: a endodontia é o ramo da odontologia que se ocupa do estudo da morfologia, da função, das alterações da polpa dental e da região periodontal, bem como de seu tratamento. $\mathrm{O}$ sucesso em endodontia é elevado, mas a sobrevivência tem sido pouco estudada. O objetivo desta pesquisa foi determinar o tempo de sobrevivência e como os fatores demográficos e clínicos afetam a perda de dentes tratados com endodontia. Métodos: fez-se um estudo de seguimento a uma coorte com análise de sobrevivência, no qual se analisou o estado dos dentes tratados com endodontia, a partir da coleta sistemática de informação, que se extraiu dos prontuários médicos. Resultados: encontra-se uma população com um ingresso baixo e com uma educação técnica ou profissional, e com predomínio dos homens; apresenta-se uma velocidade de extração ou perda do dente maior nos que têm um nível salarial baixo, e com lesões apicais maiores de $11 \mathrm{~mm}$. Discussão: para uma população específica menor de vinte anos, os resultados clínicos são similares a pesquisas publicadas em pacientes maiores a essa idade. Conclusão: nos dentes com lesões maiores a $11 \mathrm{~mm}$, a perda dental é estatisticamente significativa.

Palavras-chave: análise multivariada, endodontia, extração dental, modelo de Cox, sobrevivência. 


\section{Introducción}

La endodoncia es la rama de la odontología que se ocupa del estudio de la morfología, la función, la salud de las lesiones, las alteraciones de la pulpa dental y la región periodontal, así como de su tratamiento; dicho tratamiento, comprende todos aquellos procedimientos dirigidos a mantener la pulpa dental o a restablecer la salud de los tejidos perirradiculares [1-3].

Debido al conocimiento sobre las ventajas de la asistencia odontológica, se ha producido un aumento en todos aquellos procedimientos que permitan conservar los dientes durante más tiempo [4]. Aunque la incidencia de caries ha disminuido en algunas partes del mundo, no ha sido eliminada, y esto, sumado a un aumento de los problemas derivados de la atrición, la abrasión, la erosión y los traumatismos, ha provocado un incremento en la demanda de restauraciones fijas, con el objeto de restablecer la estética y la función dentales. Dado que los tratamientos restauradores pueden dañar la pulpa, esto conlleva igualmente a un aumento de los problemas relacionados con el complejo dentinopulpar y los tejidos perrirradiculares $[5,6]$.

El éxito en los tratamientos depende de si se alcanzan los objetivos mecánicos y biológicos, es decir, una eficiente instrumentación y obturación de los conductos, además de una adecuada restauración posterior $[7,8]$.Tal condición se mide en un rango entre 39 y $96 \%$, sólo verificable a largo plazo, y como desventaja cada examinador puede influir en los resultados [9].

Diferentes investigaciones han evidenciado que la caries, la edad, el sexo, el nivel socioeconómico, la zona de residencia (urbana y rural) y la escolaridad se asocian con la pérdida de dientes [10, 11], y dado que la salud oral no ha sido promovida adecuadamente por los gobiernos, esto genera desconocimiento de las enfermedades odontológicas que derivan en complicaciones que afectan la salud general de las personas [11-13].

En Colombia, al momento de escribir este artículo, no se encuentran estudios en revistas indexadas que hayan evaluado factores demográficos y la sobrevida del diente después de un procedimiento de endodoncia. Con esta aproximación se pretende mejorar el conocimiento de los problemas odontológicos de la población.

\section{Métodos}

Desde un enfoque empírico-analítico se realizó un estudio para asociar factores sociodemográficos y clínicos con la supervivencia del diente posterior a procedimiento de endodoncia. Se hizo un estudio de seguimiento a una cohorte con análisis de supervivencia en el que se analizó el estado de los dientes tratados con endodoncia, a partir de la recolección sistemática de información, con base en las historias clínicas. Se seleccionó el periodo 2006 hasta el 2012. La población la constituyeron los registros dentales de pacientes que se atendieron en un centro de remisión para endodoncia de Medellín durante el periodo 2006 al 2012. Entre los criterios de exclusión estuvieron los registros de pacientes con enfermedades sistémicas, no controladas, con enfermedad periodontal primaria con dientes que requieran retratamientos endodónticos. Se tomó toda la población de pacientes menores de 20 años a quienes se les realizó procedimiento de endodoncia.

Se recolectó la información en una hoja de cálculo de Microsoft Excel $^{\circledR}$ con un formato predefinido, teniendo en cuenta cada una de las variables y clasificando la información según las variables de la investigación. Esta información fue diligenciada por el(la) endodoncista que atendió al paciente, y fue registrada en la historia clínica. Se descartaron aquellas historias que no contenían la información completa o coherente con respecto a las variables de interés.

En el análisis descriptivo se usaron frecuencias absolutas, relativas y medidas de resumen. Se utilizó la prueba Chi cuadrado de independencia y la prueba exacta de Fisher para establecer asociación entre la variable dependiente, que fue la pérdida del diente, y las variables cualitativas, sociodemográficas y clínicas, que serán las variables independientes. Además, se calcularon medidas de asociación (or crudos), con sus respectivos intervalos de confianza del 95\% (IC 95\%).

La supervivencia global se calculó utilizando el análisis de Kaplan-Meier, y las curvas se compararon a través del Log Rank Test. Para el análisis multivariado se realizó el modelo de riesgos proporcionales de Cox, con fines explicativos, del cual se obtuvieron las medidas de asociación (HR) crudas y ajustadas por aquellas variables que en el análisis bivariado presentaron un valor $p$ inferior a 0,25 , siguiendo el criterio 
de Hosmer-Lemeshow. Se consideró como censura a aquellos pacientes que no presentaron el evento de interés (extracción del diente) al finalizar el estudio, y como variable de tiempo los meses transcurridos desde que se realizó el tratamiento endodóntico hasta la extracción o hasta el cierre del seguimiento. La sistematización, el procesamiento y análisis de los datos se realizaron mediante el programa spss versión 21.

Por tratarse de datos reportados en la historia clínica, la investigación se clasifica de acuerdo con la Resolución 8430 de 1993 del Ministerio de Salud, titulo II, artículo 11, como una investigación sin riesgos biológicos, fisiológicos, psicológicos y sociales para los pacientes.

\section{Resultados}

El estudio fue realizado con datos de las historias clínicas de pacientes a quienes les fue realizada endodoncia, en una IPs privada de la ciudad. La población total del estudio fue de 88 pacientes. La edad promedio de los pacientes fue de 13,63 (DE 3,3) años; el 50\% de los pacientes tenía una edad de 13,5 años o menos, con una variabilidad de 5 años, siendo 6 años la edad mínima. El sexo predominante en este grupo poblacional fue el masculino, con el 53,4\% (47), con respecto al nivel socioeconómico. Se aprecia que el 95,5\% (84) está en la categoría de menos de dos salarios mínimos legales vigentes. La ocupación más frecuente en este grupo de edad fue estudiante, con un $44,3 \%$. En cuanto a las condiciones dentales de los pacientes, se encontró que el $17 \%$ (15) tuvo pérdida dental. El diagnóstico dental más frecuente fue la periodontitis apical crónica, con el 64,4\% (56). El tipo de diente que predominó en este análisis fueron los dientes posteriores, con un $86,4 \%$.

Durante el periodo de seguimiento, el 44,3\% (39) y el $36,4 \%$ (22) de los pacientes asistieron al especialista (endodoncista) una y dos citas, respectivamente. Con respecto al tamaño de la lesión, se encontró que el 94,3\% (83) de los pacientes presentó una lesión de $10 \mathrm{~mm}$ o menos (tabla 1 ).

Tabla 1. Distribución absoluta y porcentual de factores demográficos y clínicos

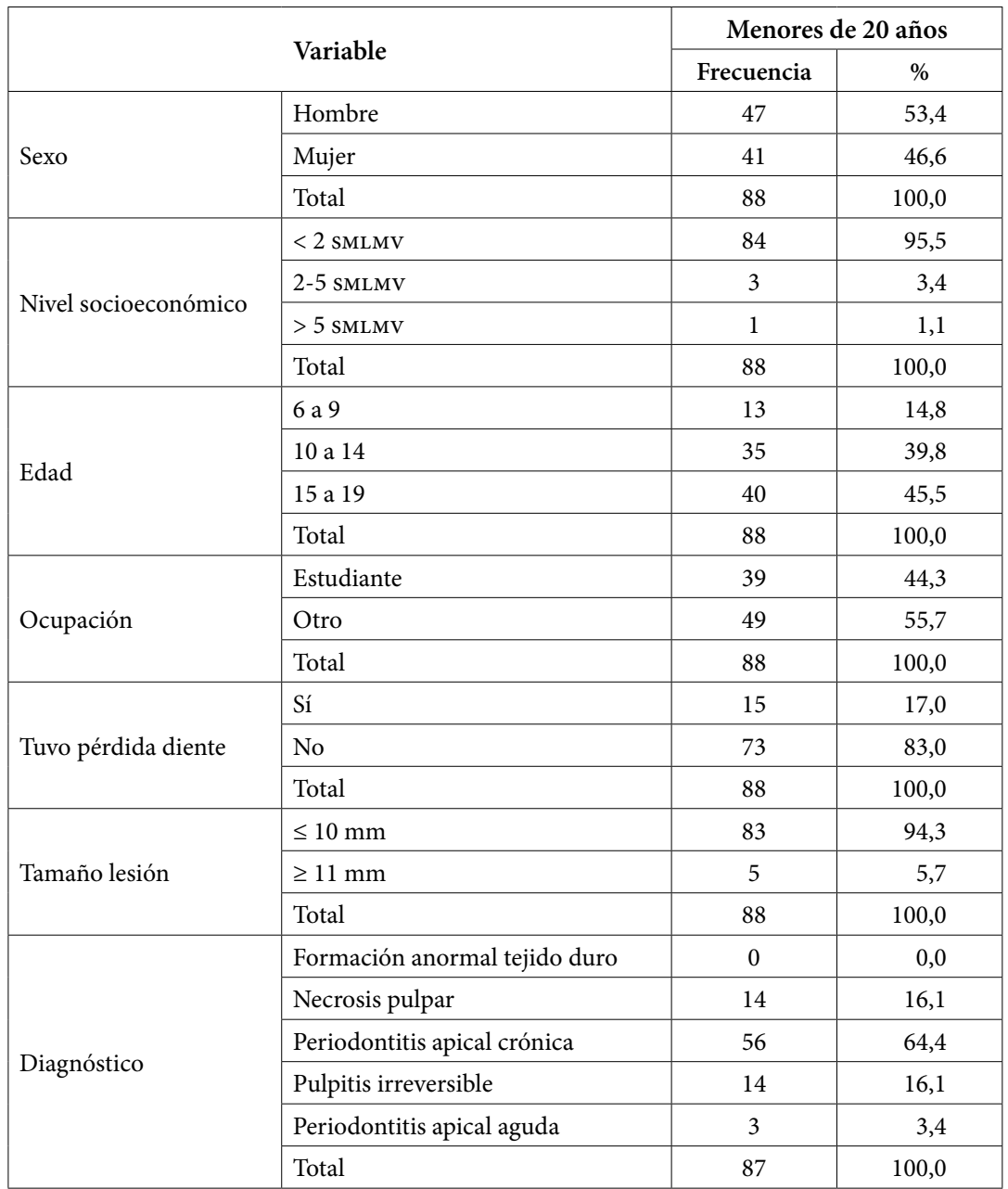




Viene
\begin{tabular}{|l|l|c|c|}
\hline \multirow{4}{*}{ Variable } & \multicolumn{2}{|c|}{ Menores de 20 años } \\
\cline { 2 - 4 } & & Frecuencia & $\%$ \\
\hline \multirow{4}{*}{ Número de citas } & 1 & 39 & 44,30 \\
\cline { 2 - 4 } & 2 & 22 & 36,40 \\
\cline { 2 - 4 } & 3 & 11 & 12,50 \\
\cline { 2 - 4 } & 4 & 6 & 6,80 \\
\cline { 2 - 4 } & Total & 88 & 100,0 \\
\hline \multirow{3}{*}{ Tipo de dientes } & Anteriores & 12 & 13,6 \\
\cline { 2 - 4 } & Posteriores & 76 & 86,4 \\
\cline { 2 - 4 } & Total & 88 & 100,0 \\
\hline
\end{tabular}

Fuente: elaboración propia

Al realizar el análisis para estudiar las posibles asociaciones de las variables de interés con la pérdida del diente por grupos de edad, se encontró que para los menores de veinte años la única variable que presen- tó asociación fue tamaño de la lesión (valor $\mathrm{p}<0,05$ ), en la que los pacientes con una lesión mayor o igual a $11 \mathrm{~mm}$ presentan 8,88 veces la oportunidad de riesgo de perder el diente (tabla 2).

Tabla 2. Distribución absoluta y porcentual de factores demográficos y clínicos versus pérdida de diente, or e intervalos de confianza

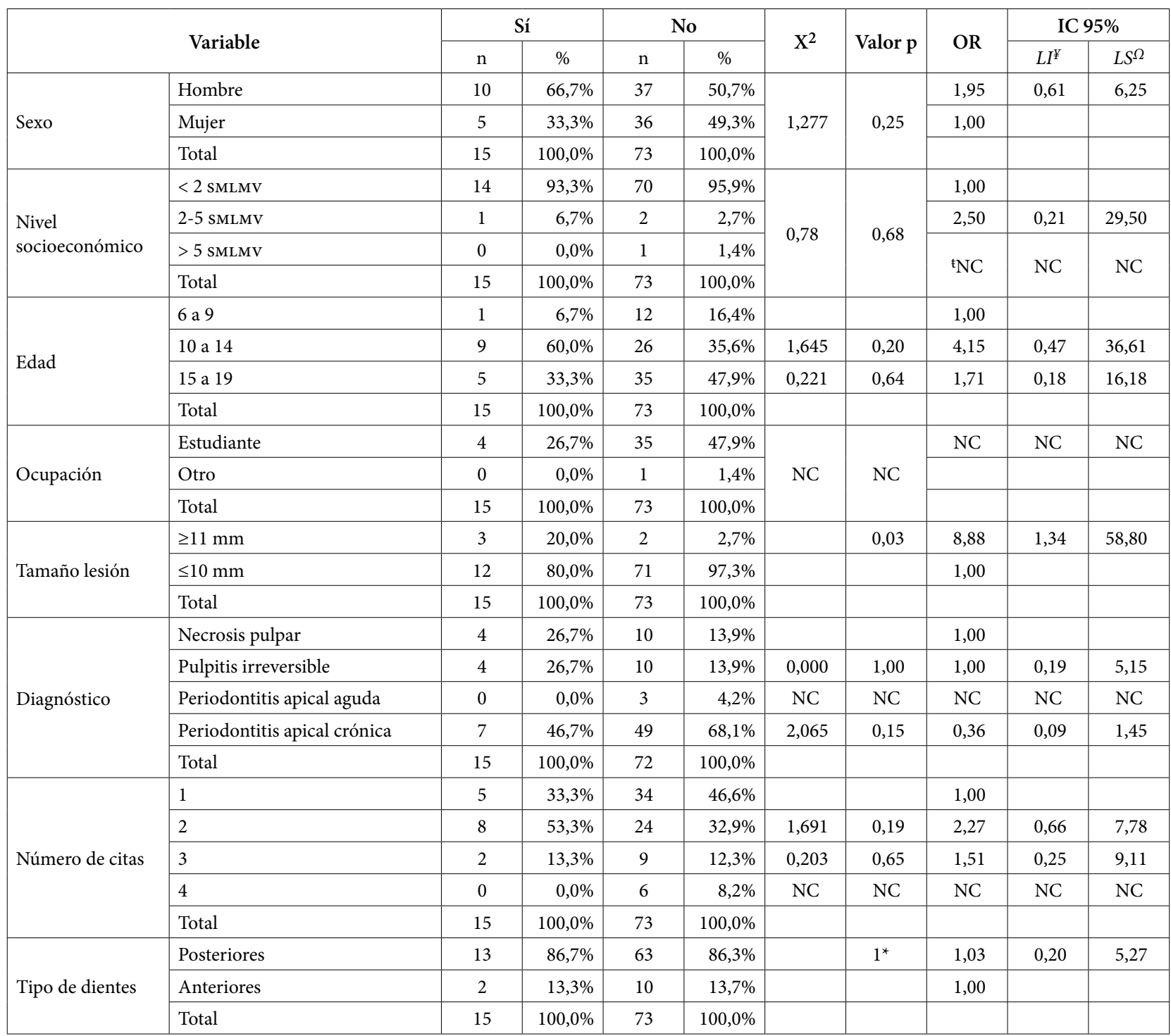




\section{Análisis de supervivencia}

En el análisis de supervivencia Kaplan-Meier se aprecia que la pérdida del diente se empieza a apreciar a partir del decimosegundo mes, y la probabilidad de no perder el diente hasta el mes 36 es del $80,4 \%$; la media de sobrevida de los dientes es de 70,58 meses (figura 1).

Al comparar el tiempo libre de pérdida del diente con las variables de interés en el estudio: sexo, edad, nivel socioeconómico, tamaño lesión, diagnóstico, número de citas y tipo de diente, la única variable que presentó significancia estadística fue tamaño de la lesión (tabla 3).

De acuerdo con el modelo, los pacientes con lesión igual o mayor a $11 \mathrm{~mm}$, la velocidad con la que ocurrió la pérdida del diente fue 7,79 veces superior respecto a lesiones menores de $10 \mathrm{~mm}$; ajustando por las demás variables del modelo la velocidad de la pérdida del diente, fue de 13,67 superior respecto a lesiones menores, siendo esta diferencia estadísticamente significativa (HR 13,267, IC 95\% 2,6-71,53) (tabla 4).

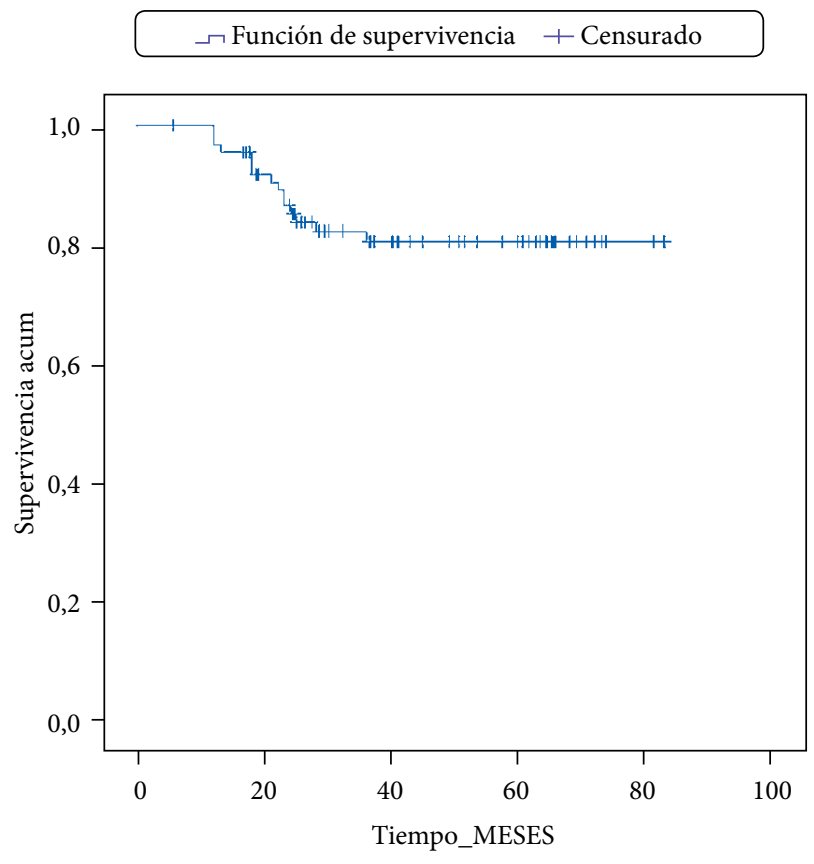

Figura 1. Función de supervivencia menores de 20 años Fuente: elaboración propia

Tabla 3. Medias de supervivencia y pruebas de significación

\begin{tabular}{|c|c|c|c|c|c|c|c|}
\hline \multicolumn{2}{|r|}{ Variable } & Estimación & Error & LI & LS & Log Rank & Valor $\mathrm{P}$ \\
\hline \multirow{2}{*}{ Sexo } & Hombre & 67,77 & 4,14 & 59,66 & 75,88 & \multirow{2}{*}{1,168} & \multirow{2}{*}{0,280} \\
\hline & Mujer & 73,90 & 3,55 & 66,95 & 80,85 & & \\
\hline \multirow{3}{*}{ Edad } & 6 a 9 & 77,65 & 4,76 & 68,33 & 86,97 & \multirow{3}{*}{3,135} & \multirow{3}{*}{0,209} \\
\hline & 10 a 14 & 57,31 & 4,34 & 48,80 & 65,82 & & \\
\hline & 15 a 19 & 73,56 & 3,69 & 66,33 & 80,80 & & \\
\hline \multirow{3}{*}{ Nivel económico } & $<2$ SMLMV & ind & ind & ind & ind & \multirow{3}{*}{1,933} & \multirow{3}{*}{0,380} \\
\hline & 2-5 SMLMV & ind & ind & ind & ind & & \\
\hline & $>5$ SMLMV & ind & ind & ind & ind & & \\
\hline \multirow{2}{*}{ Tamaño lesión } & $\geq 11 \mathrm{~mm}$ & 20,21 & 2,10 & 16,09 & 24,33 & \multirow{2}{*}{13,140} & \multirow{2}{*}{0,000} \\
\hline & $\leq 10 \mathrm{~mm}$ & 72,44 & 2,70 & 67,15 & 77,73 & & \\
\hline \multirow{4}{*}{ Diagnóstico } & Necrosis pulpar & ind & ind & ind & ind & \multirow{4}{*}{2,178} & \multirow{4}{*}{0,536} \\
\hline & Pulpitis irreversible & ind & ind & ind & ind & & \\
\hline & Periodontitis apical aguda & ind & ind & ind & ind & & \\
\hline & Periodontitis apical crónica & ind & ind & ind & ind & & \\
\hline \multirow{4}{*}{ Número de citas } & 1 & ind & ind & ind & ind & \multirow{4}{*}{3,519} & \multirow{4}{*}{0,318} \\
\hline & 2 & ind & ind & ind & ind & & \\
\hline & 3 & ind & ind & ind & ind & & \\
\hline & 4 & ind & ind & ind & ind & & \\
\hline \multirow{2}{*}{ Tipo de dientes } & Posteriores & 70,28 & 3,09 & 64,22 & 76,33 & \multirow[t]{2}{*}{0,042} & \multirow{2}{*}{0,837} \\
\hline & Anteriores & 55,96 & 4,59 & 46,97 & 64,96 & & \\
\hline
\end{tabular}

${ }^{*} \mathrm{p}<0,05$, Ind: indeterminado

Fuente: elaboración propia 
Tabla 4. Modelo de regresión de Cox múltiple

\begin{tabular}{|c|c|c|c|c|c|c|c|}
\hline \multicolumn{2}{|c|}{ Variables independientes } & \multirow{2}{*}{$\begin{array}{c}\text { HR crudo } \\
1,79\end{array}$} & \multirow{2}{*}{$\begin{array}{c}\text { IC } 95 \% \\
\text { inferior }\end{array}$} & \multirow{2}{*}{$\begin{array}{c}\text { IC } 95 \% \\
\text { superior }\end{array}$} & \multirow{2}{*}{$\begin{array}{c}\text { HR ajustado } \\
1,33 \\
\end{array}$} & \multirow{2}{*}{$\begin{array}{c}\text { IC 95\% } \\
\text { inferior }\end{array}$} & \multirow{2}{*}{$\begin{array}{c}\begin{array}{c}\text { IC } 95 \% \\
\text { superior }\end{array} \\
4,64\end{array}$} \\
\hline $\mathrm{S}_{-}$ & Hombre & & & & & & \\
\hline $\operatorname{sex}$ & Mujer & 1,00 & & & 1,00 & & \\
\hline \multirow{3}{*}{ Edad } & 6 a 9 & 1,00 & & & 1,00 & & \\
\hline & 10 a 14 & 3,83 & 0,49 & 30,28 & 4,02 & 0,47 & 33,98 \\
\hline & 15 a 19 & 1,83 & 0,21 & 15,64 & 2,06 & 0,21 & 20,45 \\
\hline \multirow{3}{*}{ Nivel económico } & $<2$ SMLMV & 1,00 & & & 1,00 & & \\
\hline & 2-5 SMLMV & 3,52 & 0,46 & 27,05 & 3,62 & 0,26 & 50,14 \\
\hline & $>5$ SMLMV & 0,00 & 0,00 & 0,00 & 0,00 & 0,00 & 0,00 \\
\hline \multirow{2}{*}{ Tamaño Lesión } & $\geq 11 \mathrm{~mm}$ & 7,79 & 2,07 & 29,31 & 13,67 & 2,61 & 71,53 \\
\hline & $\leq 10 \mathrm{~mm}$ & 1,00 & & & 1,00 & & \\
\hline \multirow{4}{*}{ Diagnóstico } & Necrosis pulpar & 1,00 & & & 1,00 & & \\
\hline & Pulpitis irreversible & 1,02 & 0,26 & 4,09 & 1,18 & 0,22 & 6,25 \\
\hline & Periodontitis apical aguda & 0,00 & 0,00 & 0,00 & 0,00 & 0,00 & 0,00 \\
\hline & Periodontitis apical crónica & 0,55 & 0,16 & 1,89 & 0,37 & 0,08 & 1,70 \\
\hline \multirow{4}{*}{ Número de citas } & 1 & 1,00 & & & 1,00 & & \\
\hline & 2 & 2,10 & 0,69 & 6,44 & 3,05 & 0,89 & 10,41 \\
\hline & 3 & 1,23 & 0,24 & 6,37 & 1,69 & 0,24 & 11,96 \\
\hline & 4 & 0,00 & 0,00 & 0,00 & 0,00 & 0,00 & 0,00 \\
\hline \multirow{2}{*}{ Tipo de dientes } & Posteriores & 1,17 & 0,26 & 5,18 & 1,67 & 0,34 & 8,29 \\
\hline & Anteriores & 1,00 & & & 1,00 & & \\
\hline
\end{tabular}

Fuente: elaboración propia

\section{Discusión}

Para analizar el éxito del tratamiento de endodoncia, este debe enfocarse desde el punto de vista biológico y clínico. Desde el punto de vista biológico, el éxito se caracteriza por la reparación tisular, y desde el punto de vista clínico, cuando después del tratamiento no se presentan signos o síntomas de infección o inflamación apical $[8,14,15]$. La regeneración lleva a que se mantenga el diente en boca por largo tiempo, pero esto va a depender también del manejo posterior, es decir, de la restauración coronal; así que cuando esta es inadecuada, pone en peligro la integridad del diente, favoreciendo la fractura y la filtración que son el origen de la pérdida [15-20]. Es el aspecto clínico mencionado y sus variables las que miden los estudios de éxito y fracaso en el tratamiento, los cuales son numerosos en la literatura [18, 21-23].

En esta investigación se consideró en el aspecto sociodemográfico el sexo y la edad, y se halló que son los hombres la población más consultante: $61,7 \%$ (500); en cuanto a la edad, se observa una edad promedio de 13 años, con predominio de población que recibe menos de dos salarios mínimos como ingreso familiar, y en su mayoría estudiantes. Esas variables caracterizan la po- blación, y no se ha hecho ningún otro estudio de supervivencia, seguramente porque no se le da importancia al esquema de seguridad social de cada país, al desestimar que la capacidad económica puede tener efecto en el tratamiento y, por tanto, en la permanencia de los dientes en la cavidad oral.

En la caracterización clínica se observa mayor prevalencia de diagnóstico de periodontitis apical asintomática, pero con tamaño de lesión inferior a $10 \mathrm{~mm}$ y atendidos en una sola cita, para un tratamiento en un diente posterior con una pérdida dental o extracción general de $17 \%$ para toda la población.

En cuanto a las asociaciones, cabe destacar que la extracción dental varía con la edad del paciente y entre los sexos, factores que a su vez generan influencia en la presencia de caries y de enfermedad periodontal, causas importantes de la pérdida dental [24]. Siguiendo con el aspecto clínico se encuentra que ser atendido en dos citas para una endodoncia de un diente posterior lleva a una mayor pérdida dental, pero con una diferencia significativa se encuentra que sí existe un riesgo mayor de pérdida en los pacientes con tamaño de lesión superior a $11 \mathrm{~mm}(12,18$ veces la posibilidad de 
riesgo de perder el diente respecto a pacientes con lesiones menores), similar a lo encontrado en la literatura al respecto, tanto en evaluación de éxito como en análisis de sobrevida.

Los avances en la endodoncia han aumentado el porcentaje de éxito en los tratamientos, el cual se encuentra entre un 70 y $95 \%$ cuando es realizado por especialistas, y entre 64 y 75\%, cuando lo hacen odontólogos [25, 26]; por esta razón, entre los factores pronósticos para la supervivencia después de un tratamiento endodóntico se encuentra la calificación del operador. En el presente estudio la riqueza está en analizar el resultado de un tratamiento hecho por un especialista con técnicas y materiales como magnificación, ultrasonido, MTA (mineral trióxido agregado), localizador apical, para evitar resultados diversos que se tienen cuando el profesional no es calificado.

La terapia endodóntica es un tratamiento con alta supervivencia a largo tiempo [15, 27-29], como lo encontramos en esta investigación, en la cual en el análisis univariado de supervivencia Kaplan-Meier se observa que la pérdida del diente se inicia a partir del decimosegundo mes, y la probabilidad de no perder el diente hasta el mes 36 es del 80,4\%.

Se presenta una mayor rapidez en la pérdida del diente en los hombres, siendo muy similar la sobrevida en los diferentes niveles de ingreso salarial. En cuanto al tamaño de la lesión, los pacientes con lesiones de más de $11 \mathrm{~mm}$ perdieron más rápido el diente, con una diferencia significativa respecto del grupo de lesiones inferiores a $10 \mathrm{~mm}$, sin diferencia significativa en cuanto al número de citas de tratamiento. Lo anterior está en acuerdo con los estudios llevados a cabo al respecto.

No hay reportes de seguimiento o supervivencia que muestren un análisis para una población menor de 20 años; esto con el propósito de evitar generalizaciones por tener condiciones dentales diferentes en lo que se refiere a la longitud radicular, al grosor de las paredes del conducto o, en general, a la mayor posibilidad de fractura en los dientes con formación incompleta de la raíz, que sería una característica importante de esta población.

En este estudio se destaca que es una población en la que tiene predominio el sexo masculino. La edad predominante en este grupo poblacional fue de 15 a 19 años, y la ocupación más frecuente en este grupo de edad fue estudiante, con un nivel de ingreso bajo de su acudiente como características sociales.
En lo relativo a las condiciones dentales de los pacientes menores de veinte años, se encontró que este grupo de edad presentó una pérdida dental de $17 \%$ con tamaños de lesión menores a $10 \mathrm{~mm}$ y con diagnóstico más prevalente de periodontitis apical crónica para tratamiento en dientes posteriores.

En las asociaciones con la pérdida o extracción dental, hay un mayor riesgo de pérdida en el hombre, el cual perdió más dientes que la mujer, en la edad entre 10 a 14 años, con lesiones mayores a $11 \mathrm{~mm}$, atendidos en dos citas y para tratamiento dientes posteriores. Todo lo anterior muy similar a lo que se encuentra para la población mayor de 20 años.

En esta población menor de 20 años, se perdieron más rápidamente los dientes en los hombres, con tamaños de lesiones mayores a $11 \mathrm{~mm}$, como variables significativas, sin posibilidades de contrastar con otras investigaciones.

En Colombia este estudio es el primero en evaluar el éxito de supervivencia del diente después de una endodoncia, en pacientes menores de 20 años, y en el que se asocian factores sociodemográficos y clínicos que influyen en la mayor rapidez de pérdida o extracción dental.

\section{Conclusiones}

En los factores sociodemograficos podemos concluir que hay mayor pérdida de dientes en la población de sexo masculino, entre los 15 y 19 años, con predominio de ingreso bajo, y estudiantes.

En los factores clínicos asociados con la pérdi$\mathrm{da}$, los pacientes con lesiones mayores de $11 \mathrm{~mm}$ pierden más sus dientes que aquellos con tamaño inferior; igualmente, quienes requieren varias citas de tratamiento pierden más sus dientes que aquellos que requieren una cita de tratamiento.

La velocidad de extracción o pérdida del diente es mayor en hombres, en el grupo de 10 a 14 años, y más rápida también para los que tienen menos salario, y con lesiones apicales mayores de $11 \mathrm{~mm}$.

\section{Referencias}

[1] Stock CJR, Gulabivival K, Walker RT, Goodman JR. Atlas en color y texto de endodoncia. $2^{\mathrm{a}}$ ed. Madrid: Mosby; 2008. 
[2] Wu MK, De Gee AJ, Wesselink PR, Moorer WR. Fluid transport and bacterial penetration along root canal fillings. Int Endod J. 1993; 26(4): 203-8.

[3] Chailertvanitkul P, Saunders WP, MacKenzie D, Weetman DA. An in vitro study of the coronal leakage of two root canal sealers using an obligate anaerobe microbial marker. Int Endod J. 1996; 29(4): 249-55.

[4] Travassos RMC, Caldas FJ, De Albuquerque DS. Cohort study of endodontic therapy success. Braz Dent J. 2003; 14(2): 109-13.

[5] Fleming CH, Litaker MS, Alley LW, Eleazer PD. Comparison of classic endodontic techniques versus contemporary techniques on endodontic treatment success. J Endod. 2010; 36(3): 414-8.

[6] Ng Y-L, Mann V, Gulabivala K. A prospective study of the factors affecting outcomes of nonsurgical root canal treatment: part 1: periapical health. Int Endod J. 2011; 44(7): 583-609.

[7] Field JW, Gutmann JL, Solomon ES, Rakusin H. A clinical radiographic retrospective assessment of the success rate of single-visit root canal treatment. Int Endod J. 2004; 37(1): 70-82.

[8] Nair PN. Apical periodontitis: a dynamic encounter between root canal infection and host response. Periodontol. 2000. 1997; 13: 121-48.

[9] Steven D. Laangzeitverweildauer wurzelkanalgefulter Zahne: eine retropektive Studie uber 10 jahre. Alemania: University of Munster; 2001.

[10] Cohen S. Vías de la pulpa. Madrid: Mosby; 2002.

[11] Nair PN, Schroeder HE. Epithelial attachment at diseased human tooth-apex. J. Periodont Res. 1985; 20(3): 293-300.

[12] Stashenko P, Yu SM, Wang CY. Kinetics of immune cell and bone resorptive responses to endodontic infections. J Endod. 1992; 18(9): 422-6.

[13] Torabinejad M, Kiger RD. Experimentally induced alterations in periapical tissues of the cat. J Dent Res. 1980; 59(1): 87-96.

[14] Nair PNR. Pathogenesis of apical periodontitis and the causes of endodontic failures. Crit Rev Oral Biol Med. 2004; 15(6): 348-81.

[15] Friedman S, Mor C. The success of endodontic therapy-healing and functionality. J Calif Dent Assoc. 2004; 32(6): 493-503.

[16] Morris MF, Kirkpatrick TC, Rutledge RE, Schindler WG. Comparison of nonsurgical root canal treatment and single-tooth implants. J Endod. 2009; 35(10): 1325-30.

[17] Hannahan JP, Eleazer PD. Comparison of success of implants versus endodontically treated teeth. J Endod. 2008; 34(11): 1302-5.

[18] Gillen BM, Looney SW, Gu LS, Loushine BA, Weller $\mathrm{RN}$, Loushine RJ et al. Impact of the quality of coronal restoration versus the quality of root canal fillings on success of root canal treatment: a systematic review and meta-analysis. J Endod. 2011; 37(7): 895-902.

[19] Field JW, Gutmann JL, Solomon ES, Rakusin H. A clinical radiographic retrospective assessment of the success rate of single-visit root canal treatment. Int Endod J. 2004; 37(1): 70-82.

[20] Aquilino SA, Caplan DJ. Relationship between crown placement and the survival of endodontically treated teeth. J Prosthet Dent. 2002; 87(3): 256-63.

[21] Cvek M, Cleaton-Jones PE, Austin JC, Andreasen JO. Pulp reactions to exposure after experimental crown fractures or grinding in adult monkeys. J Endod. 1982; 8(9): 391-7.

[22] Saunders WP, Saunders EM. Coronal leakage as a cause of failure in root-canal therapy: a review. Endod Dent Traumatol. 1994; 10(3): 105-8.

[23] Madison S, Swanson K, Chiles SA. An evaluation of coronal microleakage in endodontically treated teeth. Part II. Sealer types. J Endod. 1987; 13(3): 109-12.

[24] Hawkins RJ, Jutai DK, Brothwell DJ, Locker D. Threeyear coronal caries incidence in older Canadian adults. Caries Res. 1997; 31(6): 405-10.

[25] Imura N, Pinheiro ET, Gomes BPFA, Zaia AA, Ferraz CCR, Souza-Filho FJ. The outcome of endodontic treatment: a retrospective study of 2000 cases performed by a specialist. J Endod. 2007; 33(11): 1278-82.

[26] Alley BS, Buchanan TH, Eleazer PD. Comparison of the success of root canal therapy in HIV/AIDS patients and non-infected controls. Gen Dent. 2008; 56(2): 155-7.

[27] Salehrabi R, Rotstein I. Endodontic treatment outcomes in a large patient population in the USA: an epidemiological study. J Endod. 2004; 30(12): 846-50.

[28] Swartz DB, Skidmore AE, Griffin JA Jr. Twenty years of endodontic success and failure. J Endod. 1983; 9(5): 198-202.

[29] Dammaschke T, Steven D, Kaup M, Ott KHR. Longterm survival of root-canal-treated teeth: a retrospective study over 10 years. J Endod. 2003; 29(10): 638-43. 